\title{
¿SALIR O SALIR CORRIENDO? UNA APROXIMACIÓN A LA CONSTRUCCIÓN VERBO + GERUNDIO DE MODO EN TEXTOS ORIGINALES Y TEXTOS TRADUCIDOS AL ESPAÑOL
}

doi.org/10.15452/SR.2021.21.0009

ORCID ID: 0000-0002-6392-8741

\author{
Petr Šlechta \\ Universidad de Ostrava, \\ República Checa \\ petr.slechta@osu.cz
}

Resumen. Este artículo se dedica al gerundio de modo en combinación con verbos de movimiento. El punto de partida de este estudio es el marco teórico propuesto por la semántica cognitiva que sostiene que un evento de movimiento se puede dividir en varios componentes: MOVIMIENTO, TRAYECTORIA, FIGURA y BASE. Con respecto a los patrones de lexicalización predominantes se distinguen dos tipos de lenguas: lenguas de marco satélite (que codifican la TRAYECTORIA por medio de un «satélite») y lenguas de marco verbal (que expresan la TRAYECTORIA mediante la raíz verbal). Además, se ha observado que los hablantes del segundo tipo prestan menos atención a la expresión de MANERA, un componente secundario, y que en situaciones de «cruce de frontera» existen restricciones importantes que afectan este componente. Para explorar el uso de gerundio en combinación con verbos de movimiento se utilizaron los corpus InterCorp y Araneum Hispanicum Maius albergados por el Instituto del Corpus Nacional Checo. Los resultados indican que el gerundio de modo se usa con mayor frecuencia en combinación con salir, ir, venir y llegar y las formas más comunes son corriendo, caminando, andando y volando. También muestran que las combinaciones con corriendo y volando son más frecuentes en el subcorpus de textos traducidos al español que en los textos originales. El autor concluye que la dinámica del evento es importante y por eso la información de MANERA se mantiene en las traducciones.

Palabras clave. Verbos de movimiento. Semántica cognitiva. Gerundio de modo. Lingüística de corpus. Traducción. 


\begin{abstract}
Salir or salir corriendo? An Approach to the Construction of verb + gerund of manner in Original Texts and Texts Translated into Spanish. This article deals with the gerund of manner in combination with verbs of motion. The starting point of this study is the theoretical framework proposed by cognitive semantics which maintains that a motion event can be divided into several components: MOTION, PATH, FIGURE and GROUND. With respect to the predominant lexicalization patterns, two types of languages are distinguished: satellite-framed languages (which encode the PATH by means of a "satellite") and verb-framed languages (which express the PATH using the verb stem). In addition, it has been observed that speakers of the second group pay less attention to the expression of MANNER, a secondary component, and that there are significant restrictions affecting this component in "boundary-crossing" events. To explore the use of the gerund in combination with verbs of motion, the InterCorp and Araneum Hispanicum Maius corpora, hosted by the Institute of the Czech National Corpus, were used. The results indicate that the gerund of manner is most often used in combination with salir, ir, venir, and llegar, and the most common forms are corriendo, caminando, andando, and volando. They also show that the combinations with corriendo and volando are more frequent in the subcorpus of texts translated into Spanish than in the subcorpus of original texts. The author concludes that the dynamics of the event is important and that is why the MANNER information is kept in the translations.
\end{abstract}

Keywords. Verbs of motion. Cognitive semantics. Gerund of manner. Corpus linguistics. Translation. 


\section{Introducción}

El presente artículo contrasta el uso del gerundio de modo o manera (cf. NGDLE) introducidos por verbos de movimiento en textos narrativos traducidos al español con su frecuencia en textos originales. Nos apoyamos principalmente en el marco teórico propuesto por la semántica cognitiva y en datos reales provenientes de los corpus paralelos InterCorp (Rosen; Vavřín; Zasina, 2020) y el corpus comparable Araneum Hispanicum Maius (Benko, 2015).

Después de una breve presentación de las pautas teóricas, vamos a centrarnos en los siguientes aspectos: a) qué verbos de movimiento suelen aparecer en combinación con el gerundio de modo o manera; b) qué gerundios de modo o manera se suelen combinar con los verbos de movimiento con mayor frecuencia; y, por último, c) con qué frecuencia aparece la estructura en cuestión en textos originales frente a textos traducidos.

\subsection{Semántica cognitiva y la expresión de movimiento}

La representación lingüística del movimiento es diferente de la descripción hecha por la física. En la semántica cognitiva se ha propuesto un modelo que denomina «eventos de movimiento» (motion events) aquellas situaciones en las que se realiza un movimiento ${ }^{1}$ (Talmy, 1985; 1991; 2000a; 2000b) y estos eventos se estructuran en los siguientes componentes: ${ }^{2}$

a) FIGURA (FIGURE): la entidad que se está moviendo;

b) BASE (GROUND): el objeto de referencia;

c) MOVIMIENTO (MOTION): el propio movimiento;

d) TRAYECTORIA (PATH): el camino que recorre la FIGURA.

En la realización lingüística, algunos componentes se suelen fusionar en un solo elemento (p. ej. salió en el ej. [1] denota tanto el MOVIMIENTO como la TRAYECTORIA). Esta estructuración es la base de la tipología semántica de lenguas que distingue dos grupos: por un lado, las lenguas de marco verbal (verb-framed languages), esto es, aquellas que predominantemente expresan la TRAYECTORIA mediante la raíz verbal y, por otro lado, las lenguas de marco satélite (satellite-framed languages), que para lo mismo utilizan un «satélite», un elemento cercano a la raíz verbal (a menudo un prefijo o una preposición).

(1) checo: Včela VYletěla $z$ úlu.

inglés: The bee flew OUT of the hive.

español: La abeja SALIÓ (volando) de la colmena.

1 Sin embargo, según Talmy (2000a: 8), un evento de movimiento puede comprender también situaciones que describen la localización de un objeto (location). Cuartero Otal (2006) ofrece un análisis crítico más detallado de los enfoques similares, enfatizando la distinción entre verbos de desplazamiento y de modo de desplazamiento.

2 Utilizamos la terminología en concordancia con Cifuentes Férez (2012), salvo con la diferencia del último componente, para el cual, al estilo de Martinková (2018) preferimos usar el término TRAYECTORIA en vez de CAMINO para evitar una posible confusión con las metáforas conceptuales de Lakoff y Johnson. 
Según Slobin (2006), el primer grupo incluye p. ej. lenguas semíticas, túrquicas, griego, coreano, japonés y también lenguas románicas, mientras que al segundo grupo pertenecen p. ej. lenguas germánicas, lenguas ugrofinesas y lenguas eslavas. ${ }^{3}$

\subsection{La restricción del cruce de frontera}

Aparte de los componentes mencionados en la sección 1.1, existen también los co-eventos (co-events, Talmy, 2000a) que tienen una función secundaria (Cifuentes Férez, 2012). Uno de los co-eventos es la MANERA que expresa la modalidad en la que se realiza el movimiento. Slobin y Hoiting (1994), apoyándose en el trabajo de Aske (1989), señalaron que en eventos en los que la FIGURA cruza una frontera (boundary crossing events), lenguas de marco verbal no pueden expresar la MANERA a través del verbo:

(2) checo: Včela vyletěla z úlu.

inglés: The bee flew out of the hive.

español: *La abeja voló de la colmena. $\rightarrow$ La abeja salió (volando) de la colmena.

En estas diferencias entre las lenguas ya se fijaron por ejemplo Vinay y Darbelnet (1958: 107), atribuyéndolas al «espíritu de la lengua» (génie de la langue); una interpretación en la misma línea la encontraríamos también en Alonso (1961: 13) quien habla del «peculiar pensamiento idiomático». Tesnière (2015: 312) compara el ejemplo del alemán, Anton schwimmt über den Fluß, con la frase correspondiente en francés, Antoine traverse le fleuve à la nage ('Antonio atraviesa nadando el río'), señalando que, mientras que en alemán los verbos expresan el movimiento, en francés lo que muestran es el desplazamiento (es decir, no se centran en la manera de movimiento). Por su parte, Slobin (2006) argumenta que la lingüística cognitiva nos facilita una explicación más precisa: para expresar la MANERA en el caso del español se tiene que hacer uso de un elemento subordinado que es más difícil de procesar cognitivamente, como, por ejemplo, un gerundio (volando en el ej. [1]) o locución adverbial (p. ej. ir a pie). No obstante, distintos autores (Cifuentes Férez; Rojo López 2015, Filipović, 2008, Slobin, 1996, 2004, 2006; entre otros) han demostrado que, a pesar de la disponibilidad de estos recursos, los hablantes de lenguas de marco verbal prestan menos atención al componente de MANERA. ${ }^{4}$ Por otro lado, lenguas de marco satélite suelen contar con un elenco muy numeroso de verbos capaces de captar diferentes matices de la manera de movimiento (Slobin, 1996).

3 La división bipartita ha sido criticada en varias ocasiones, puesto que, según distintos autores, existe variación intratipológica (p. ej. Ibarretxe, 2003) y la tipología debería ser ampliada (Croft, et al., 2010). Slobin (2004) propone la inclusión de «lenguas de marco equipolente» (equipollently-framed languages).

4 Slobin (2006) explica que la prominencia de este rasgo, entre otros aspectos, depende del repertorio de imágenes mentales de los usuarios de la lengua en cuestión, el estilo narrativo y propiedades lexicales y morfosintácticas. El mismo autor (2005: 5-7) señala que hablantes de lenguas de marco satélite tienden a centrarse más en la TRAYECTORIA (sus partes, su curso) en sus discursos, mientras que los usuarios de lenguas de marco verbal se centran en los aspectos físicos del entorno y prefieren inferir los detalles sobre ella. 


\subsection{El gerundio de modo}

El gerundio que participa en las construcciones como salir volando se suele denominar el «gerundio de modo» 0 «gerundio de manera» (cf. NGDLE). En dicha construcción, a diferencia de las estructuras denominadas «perífrasis verbales» ${ }^{6}$ en las que la combinación de un verbo principal, semánticamente más vacío y conjugable, y el verboide (forma no finita), obligatorio, invariable y con mayor peso semántico, se utiliza para aportar el significado modal o aspectual, la construcción de un verbo de movimiento en combinación con un gerundio de modo, fundamentalmente, representa dos acciones simultáneas:

(3) Juan está corriendo ( ${ }^{\star} J u a n$ está) vs. Juan sale corriendo (Juan sale [y corre/está corriendo]).

La NGDLE (3264 y ss.) nos ofrece dos opciones de cómo analizar el gerundio en la expresión salir corriendo (y casos analógicos). Por un lado, se trataría de la interpretación temporal; en concreto, sería el «gerundio de simultaneidad» en el que la acción del gerundio «se entiende como una acción simultánea con la acción o el proceso al que se refiere el verbo principal, sean estos sucesos pasados, presentes o futuros» (salir y correr). Por otro lado, la gramática menciona (3270 y ss.) la posibilidad de interpretar estos casos como «gerundio de modo o manera» (¿Cómo sale? - Corriendo). No obstante, también señala que «[1]os límites entre la interpretación temporal del gerundio y la de [modo] o [manera] son igualmente inseguros en ciertos contextos, sobre todo porque son escasos los índices gramaticales que distinguen con claridad estos valores» ${ }^{7}$ y que «[1]a interpretación de modo resulta a menudo la correcta cuando los verbos de movimiento aparecen modificados por gerundios que expresan alguna manera de moverse, como en Se acercó arrastrándose, en Entró bailando [...]. Sin embargo, cuando el gerundio que no expresa manera de moverse modifica a un verbo que denota movimiento, la frontera entre la interpretación de simultaneidad y la de modo o manera resulta casi imposible de establecer [...].»

\subsection{Consecuencias para la traducción}

Slobin (2006: 70), resumiendo sus hallazgos anteriores, menciona que, en traducciones de ficción creativa,

manner salience follows patterns of the target rather than source language. [...] translations into satellite-framed languages add manner information, whereas translations into verb-framed languages remove manner information. This is true both with regard to lexical items and more extended descriptions of manner of motion.

5 No obstante, Fernández Lagunilla (1999) en relación con este tipo de gerundios utiliza la etiqueta «gerundios lexicalizados». En efecto, el concepto de gerundio de modo es más amplio; en este texto nos dedicamos solo a un subtipo: los gerundios de verbos de manera de movimiento.

6 Como advierte p. ej. Yllera (1999: 3395-3396), no hay consenso unívoco sobre la clasificación de distintas combinaciones de verbos conjugados y gerundios, dependiendo de los criterios aplicados (sintácticos, semánticos, funcionales); según algunos autores la construcción salir + gerundio podría considerarse perifrástica, otros la situarían entre las perífrasis y las locuciones.

7 Apoyan esta idea también Nádvorníková et al. (2020: 112). Por su parte, Whitley y González (2015: 194) mencionan que el gerundio con función adverbial -que ellos denominan «gerundio de manera»- también puede designar la dirección, como en vino bajando del monte. 
En su interesante estudio, Filipović (2008) analiza transcritos de declaraciones de testigos y personas sospechosas. Es evidente que, en situaciones donde se da cuenta sobre un robo, un atraco o una escena parecida, los detalles sobre la escena pueden tener un papel trascendental. Sin embargo, una de sus conclusiones es que «Spanish speakers use manner verbs extremely rarely, and even when explicitly asked to expatiate on the manner of motion, they find it difficult». Admite que, aunque la manera de movimiento podía haber sido deducida del contexto sin ser expresada o porque fue mencionada anteriormente, los intérpretes a veces asumían que la manera de movimiento, mencionada una vez al principio del testimonio, seguía siendo la misma, lo que, como explica, no siempre tiene que ser verdad. Matinková (2018: 41), comentando dicho estudio, observa que la falta de verbos de movimiento que codifiquen la MANERA en este tipo de estudios realizados sobre el español está en un fuerte contraste con el hecho de que se trata de situaciones muy dinámicas en las que esperaríamos una gran cantidad de verbos que expresen cómo se realizó el movimiento. Filipović (2008: 29) añade que el dinamismo de la situación es importante, porque puede revelar datos cruciales sobre la velocidad del evento y p. ej. ayudar a estimar la localización de la FIGURA en cuestión, en relación con la distancia desde el punto de partida (por ejemplo, en la escena de un crimen).

Sintetizando lo expuesto anteriormente, el equivalente más natural de la frase en checo del ejemplo (1) sería la solución sin el gerundio volando (La abeja salió de la colmena); además, en la traducción al español de una lengua de marco satélite podemos esperar que se pierda la información sobre la MANERA, mientras que en la traducción del español a una lengua de marco satélite es probable (si no inevitable) que esta sea añadida.

\section{Metodología}

Primero, para examinar qué verbos principales participan más a menudo en la estructura en cuestión hemos aprovechado el acceso al corpus Araneum Hispanicum Maius (Benko, 2015). ${ }^{8}$ Como, en general, la estructura compuesta por un verbo y gerundio representa una gama muy amplia y variada de ejemplos de los que la mayoría no nos interesa en este momento, ${ }^{9}$ hemos elegido cuatro gerundios que, con más probabilidad, podrían tener la interpretación de modo o manera: corriendo, caminando, andando y volando.$^{10}$ Recuperadas las concordancias, procedimos al filtrado del contexto izquierdo según la posición IL desde el KWIC (key word in context,

8 Accesible desde www.korpus.cz. Se trata de un corpus no referencial, no representativo y comparable con un tamaño de 1200000617 tokens. Está basado en textos provenientes de páginas web en español. Elegimos este corpus ante todo por las posibilidades de consulta avanzadas que ofrece la interfaz Kontext a través de la cual se accede (listados de lemas con frecuencias, filtros, consultas CQL, etc.).

9 Por ejemplo, si utilizáramos la consulta CQL [tag=“V.*”][word=“.*ndo”Etag=“V.*”]within <s/>, es decir, «busca todos los lemas verbales seguidos inmediatamente por todas las formas terminadas en -ndo que a la vez son verbos y están dentro de la misma oración» (1963140 tokens, i. p. m. 1635,95), recuperaríamos ejemplos de dicha estructura, pero la mayoría serían perífrasis verbales con gerundio (estar paseando, ir yendo, continuar perdiendo, etc.).

10 Semánticamente se refieren a modalidades básicas de movimiento de entidades vivas. La consulta para el caso del gerundio corriendo fue [word=“corriendo"] y analógicamente procedimos en los demás casos. 
'palabra clave en contexto') y generamos la lista de lemas ordenada por frecuencia. De ella, escogimos manualmente cinco verbos de movimiento (desplazamiento) más frecuentes utilizados como verbo principal.

Por otro lado, para identificar gerundios de qué verbos son los más comunes en la estructura en cuestión, escogimos los verbos principales ir, venir, volver, llegar, salir, entrar y viajar y en el mismo corpus aplicamos la consulta [lemma="ir"] [word=“.*ndo" $\&$ tag="V.*”] para recuperar los ejemplos en los que el lema ir va seguido directamente por una forma verbal terminada en -ndo y analógicamente procedimos en los demás casos. Generamos la lista de lemas y manualmente escogimos aquellos casos que podrían ser considerados gerundios de modo."

Por último, para averiguar si la estructura en cuestión tiene la misma incidencia en el lenguaje de las traducciones como en textos originales, utilizamos el segmento nuclear de Inter$\operatorname{Corp}^{12}$ (Čermák; Vavřín, 2020), creando un subcorpus de textos originales en español (12 225678 posiciones) y otro de textos no originales en español ${ }^{13}$ (19008361 posiciones). De ellos, recuperamos las ocurrencias de combinaciones de los verbos principales más comunes (salir, ir, venir y llegar), junto con los gerundios de modo caminando, corriendo y volando. ${ }^{14}$ Como estamos comparando ocurrencias entre dos subcorpus con un tamaño diferente, trabajamos con la frecuencia relativa. Para distinguir si las diferentes frecuencias de uso de las expresiones tienen significancia estadística o son producto de azar, calculamos el valor $p \cdot{ }^{15}$ Además, añadimos los valores del corpus CREA para contrastar la información obtenida de InterCorp con un corpus más equilibrado.

\section{Resultados}

\subsection{El verbo principal en la construcción verbo de movimiento + gerundio}

11 Tenemos que confirmar lo que señala la NGDLE: decidir si se trata de un gerundio de modo es problemático. Nos guiamos por la prueba mediante la pregunta ¿Cómo? para escoger aquellos casos que eran semánticamente más relevantes como respuestas a esa pregunta.

12 InterCorp es un conjunto de corpus referenciales, no representativos y paralelos disponible en www. korpus.cz. En total cuenta con 41 lenguas, siendo el checo la lengua pivote (para más información sobre el corpus véase https://wiki.korpus.cz/doku.php/en:cnk:intercorp:verzel3). El segmento nuclear (Core) contiene textos literarios que fueron alienados manualmente con los textos traducidos, así que ofrece una mayor precisión que los otros segmentos (Aquis, EuroParl, Subtitle, etc.) que fueron procesados automáticamente.

13 Hablamos de textos traducidos al español sin diferenciar cuál fue la lengua origen, puesto que lo que nos interesa es observar el lenguaje de las traducciones en general. Las lenguas fuente más representadas son checo, inglés, alemán y francés.

14 La consulta para salir caminando fue [lemma="salir"][word="caminando"] y analógicamente procedimos en los demás casos probados.

15 Para calcular el valor $p$ utilizamos la herramienta denominada «Calc» (Cvrček, 2019) disponible en la propia interfaz de los corpus ofrecidos por el Instituto del Corpus Nacional Checo en https://www. korpus.cz/calc/. La herramienta cuenta con el nivel de significación $\alpha$ igual a 0,05; es decir, los valores $p$ superiores a este número indican que las diferencias entre las frecuencias relativas comparadas no son estadísticamente significativas y, al contrario, los valores inferiores indican que la diferencia entre las frecuencias relativas es estadísticamente significativa. 
Los datos muestran que en la construcción tratada aquí participan con mayor frecuencia como verbos principales los verbos salir, ir, venir y llegar.

\begin{tabular}{|l|l|}
\hline Gerundio & Verbos principales más comunes \\
\hline corriendo & salir (3914),16 ir (1828), venir (344), llegar (162), bajar (162) \\
\hline caminando & ir (1393), llegar (161), venir (149), salir (105), volver (53) \\
\hline andando & ir (770), subir (75), llegar (75), venir (58), salir (49) \\
\hline volando & pasar (806), ${ }^{17}$ salir (649), ir (316), venir (64), llegar (51) \\
\hline
\end{tabular}

Tabla 1: Los verbos principales más frecuentes

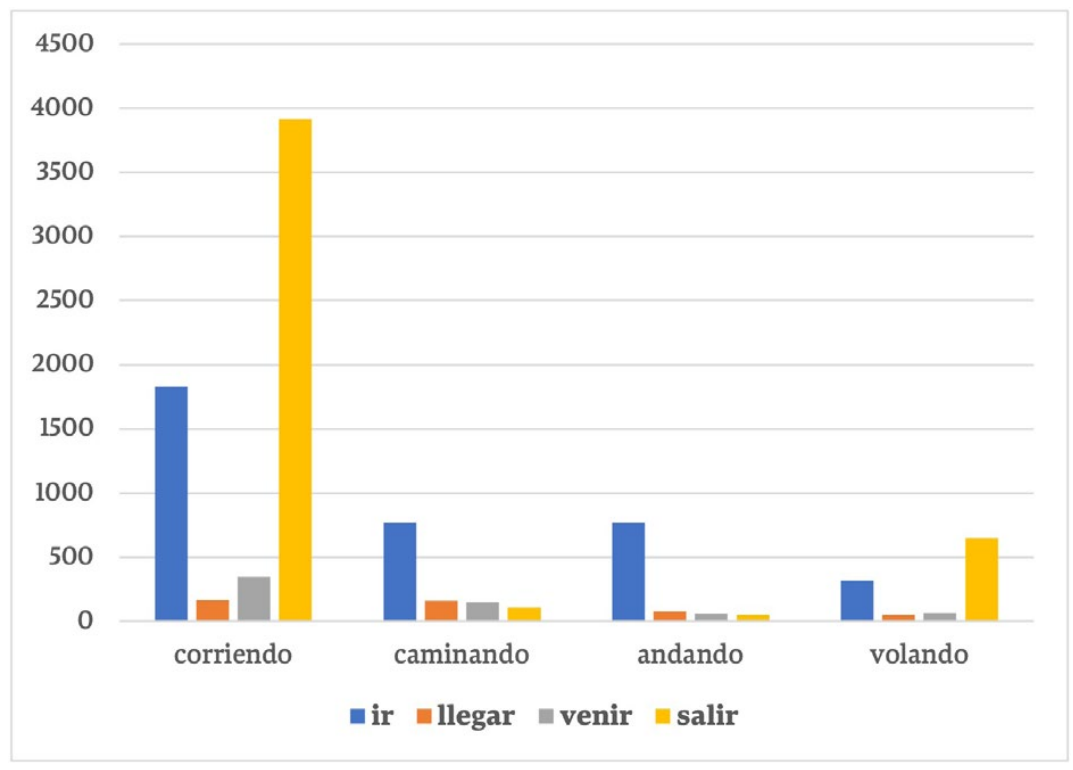

Fig. 1 : Los verbos principales más frecuentes

\subsection{Los gerundios de modo en la estructura verbo + gerundio}

Los gerundios de modo más representados en el corpus fueron corriendo, caminando, andando y volando, tal como muestra la Tabla 2.

16 Los números entre paréntesis indican la frecuencia absoluta. En el procesamiento automático de datos que se utiliza en la creación de los corpus, la adscripción de lemas y la desambiguación no siempre fueron correctas, por lo tanto, en los casos de salir (que también fue lematizado como salar por las formas idénticas de indicativo de subjuntivo) e ir (algunas de cuyas formas coinciden con ser), el número representa la suma de todas las frecuencias. En esta parte, además, solo trabajamos con listas de frecuencias sin entrar en las concordancias. Los datos, en consecuencia, no están completamente «limpios» porque pueden contener unidades fraseológicas o perífrasis verbales que no son de nuestro interés.

17 Mediante la consulta adicional [lemma="tiempo"][] $\{0,5\}[$ lemma="pasar"][word="volando"] podemos descubrir que 214 de las ocurrencias de pasar volando forman parte de la frase proverbial El tiempo pasa volando. 


\begin{tabular}{|l|l|}
\hline $\begin{array}{l}\text { Verbo } \\
\text { principal }\end{array}$ & Gerundios de modo más comunes \\
\hline ir & corriendo (1571), caminando (1277), cayendo ${ }^{18}$ (1084), andando (713), volando (323) \\
\hline venir & $\begin{array}{l}\text { corriendo (345), arrastrando (276), pisando } \\
\text { (139) (193), caminando (149), manejando }\end{array}$ \\
\hline volver & corriendo (100), caminando (53), andando (47), volando (13), pisando (12) \\
\hline llegar & corriendo (162), caminando (161), andando (75), volando (52), pisando (41) \\
\hline viajar20 & volando (4), andando (3), conduciendo (2), huyendo (2), pedaleando (1) \\
\hline salir & corriendo (3538), volando (594), huyendo (435) , caminando (105), andando (49) \\
\hline entrar & corriendo (128), caminando (23), volando (18), pisando (17), andando (9) \\
\hline
\end{tabular}

Tabla 2. Los gerundios de modos más frecuentes

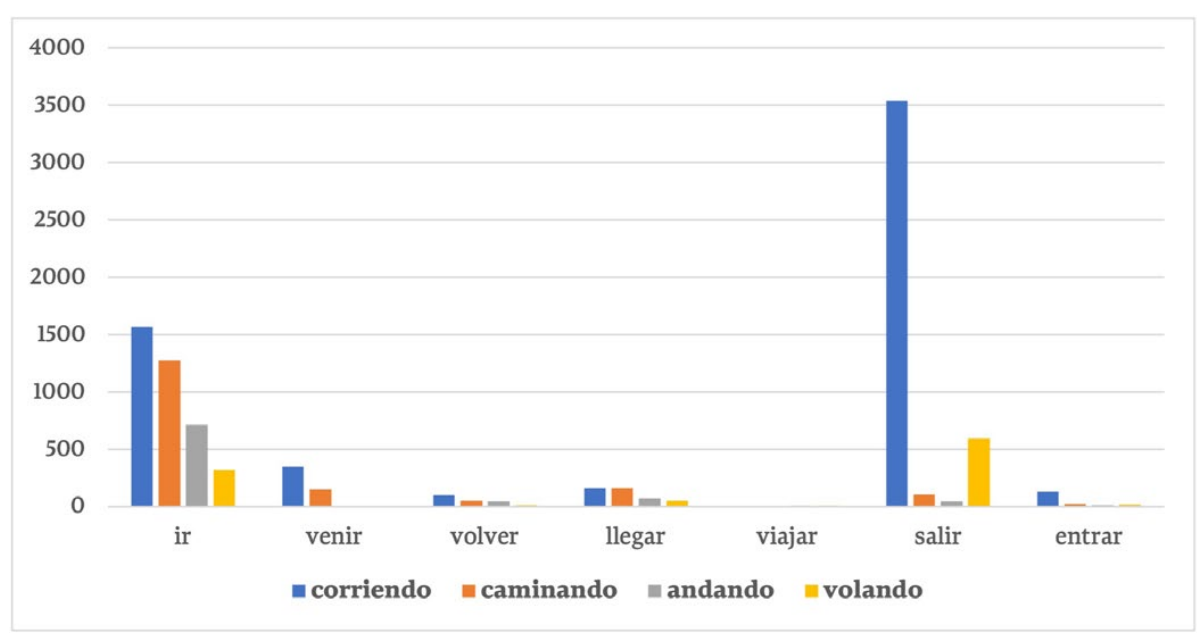

Fig. 2 : Los gerundios de modo más frecuentes

\subsection{Textos originales vs. textos traducidos}

Los datos muestran que, en la mayoría de los casos probados, las traducciones son más explícitas en cuanto a la manera de movimiento expresada por el gerundio de modo, en los ejemplos

18 Muchos de los casos forman parte de la perífrasis durativa ir cayendo, por lo cual, si realizáramos una refinación de estos datos, el número sería menor.

19 Este es un caso especial porque necesita complementación. Filtrando el contexto derecho de las concordancias hemos descubierto que 177 de las ocurrencias formaban parte de la locución verbal pisar fuerte. En las otras ocurrencias de pisando (en combinación con volver, llegar y entrar) también prevalece el uso de dicha locución verbal.

20 El repertorio de gerundios de modo que coaparecen con el verbo viajar es diferente, puesto que el significado de este verbo es más concreto y su compatibilidad semántica con ellos es limitada (p. ej. casos de viajar corriendo en los corpus disponibles no aparecen, pero sí podemos documentar casos esporádicos de esta expresión en distintas páginas web dedicadas a un fenómeno nuevo, los running tours, en los que adquiere el significado literal).

21 Recuperamos también 202 ocurrencias de salir pitando, pero, como se trata de una expresión figurativa en la cual el verbo pitar pierde su significado original, no la hemos incluido en la lista. 
de salir corriendo y salir volando de forma significativa, igual que en el caso de ir corriendo, llegar corriendo y llegar volando. Lo contrario ocurre con los ejemplos en los que figura el gerundio caminando, pues aquellas combinaciones son más comunes en textos originales (no obstante, solo en el caso de salir caminando se da de forma significativa).

\begin{tabular}{|l|l|l|l|l|l|}
\hline Estructura & CREA & $\begin{array}{l}\text { Textos } \\
\text { originales }\end{array}$ & $\begin{array}{l}\text { Textos } \\
\text { traducidos }\end{array}$ & Valor $\mathbf{p}$ & Significación \\
\hline salir caminando & $0,22^{22}$ & 3,19 & 0,37 & 0 & + \\
\hline salir corriendo & 6,19 & 12,19 & 21,15 & 0 & + \\
\hline salir volando & 0,68 & 2,37 & 6,25 & 0 & + \\
\hline ir caminando & 1,67 & 2,94 & 1,89 & 0,0591 & - \\
\hline ir corriendo & 2,02 & 1,88 & 4,79 & 0 & + \\
\hline ir volando & 0,53 & 0,65 & 0,95 & 0,7652 & - \\
\hline venir caminando & 0,3 & 0,65 & 0,37 & 0,2601 & - \\
\hline venir corriendo & 0,66 & 1,72 & 2,68 & 0,0829 & - \\
\hline venir volando & 0,25 & 0,25 & 0,42 & 0,4199 & - \\
\hline llegar caminando & 0,13 & 0,16 & 0,05 & 0,3287 & - \\
\hline llegar corriendo & 0,41 & 1,31 & 3,16 & 0,0012 & + \\
\hline llegar volando & 0,1 & 0 & 1,32 & 0,0001 & + \\
\hline
\end{tabular}

Tabla 3: La construcción con gerundio de modo en textos originales y en textos traducidos al español

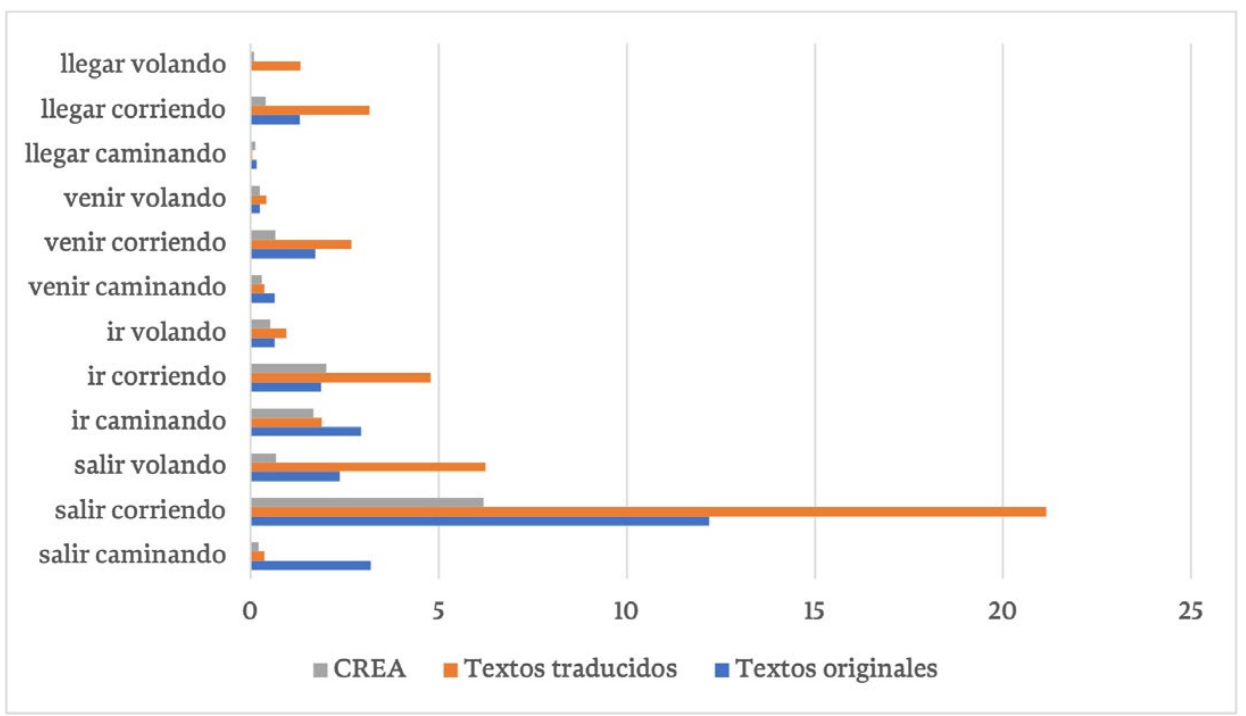

Fig. 3: La construcción con gerundio de modo en textos originales y en textos traducidos al español (i. p. m.)

22 Todas las frecuencias son relativas (instancias por millón; i. p. m.). 


\section{Discusión}

En primer lugar, cabe mencionar que las frecuencias relativas de la construcción de verbo de movimiento con gerundio de modo recuperadas de los textos originales de InterCorp no coinciden con las frecuencias relativas halladas en el CREA. Esto se debe, con mayor probabilidad, a que nuestro subcorpus de InterCorp solo contiene un elenco limitado de textos literarios, mientras que el CREA es más grande y equilibrado (aparte de obras literarias contiene también la lengua oral, artículos de prensa y otros textos).

El hallazgo que consideramos digno de atención es la diferente representación de la construcción en cuestión en textos originales y en textos traducidos, puesto que contrasta con lo que expusimos en 1.4: en las traducciones, supuestamente, debería ser menos frecuente, puesto que, como argumenta Slobin (2006), los traductores suelen guiarse por los patrones típicos en la lengua meta, en este caso español, que es una lengua de marco verbal y por eso, la manera más natural sería utilizar el verbo de movimiento sin el gerundio de modo. Sin embargo, nuestros datos muestran que la incidencia es más baja solo en los casos con el gerundio caminando (únicamente en salir caminando con significación estadística), pero en los demás casos las frecuencias en textos traducidos superan las identificadas en los textos originales (de forma significativa en los casos de salir corriendo, salir volando, ir corriendo, llegar corriendo y llegar volando). Si quisiéramos interpretar este hecho dentro del marco cognitivo, parece que no todos los componentes de MANERA gozan de la misma importancia para la percepción humana. Cuando se trata de un evento más dinámico o rápido, le prestamos más atención:

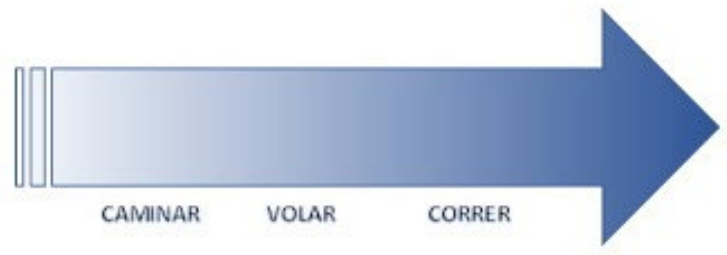

Fig. 4: Importancia del dinamismo

En términos de Talmy (2000b: 128), estaríamos ante los procesos de foregrounding y backgrounding o, dicho de otro modo, desde una terminología más tradicional, estaríamos ante la dicotomía «marcado» vs. «no marcado». Aske (1989, p. 10) señala que «manner information tends to be highly rhematic», esto es, que la información sobre la MANERA es muy marcada. A pesar de ello, nuestros datos insinúan que se trata de una cuestión de escala (o continuo), y que las acciones más dinámicas (correr, volar) son más marcadas que las menos dinámicas (caminar), lo que también se refleja en las traducciones, puesto que se mantiene un elemento no obligatorio y más difícil para el procesamiento mental (salir corriendo en vez de salir). ${ }^{23}$

23 Por supuesto, en relación con el marcaje de la MANERA, cabe considerar también la habitualidad del tipo de movimiento, no solo con respecto a la FIGURA en cuestión, sino también con el evento de 
Destaca, además, el caso de salir corriendo cuya incidencia es más de doble en los textos traducidos, aunque en los textos originales es también considerablemente más frecuente que los demás casos probados. Eso indica que podría tratarse de una unidad convencionalizada ${ }^{24}$ o institucionalizada (cf. Corpas Pastor, 1996: 21) o, simplemente, en vías de un proceso de habitualización gracias al que perdería cierto «peso» (processing load) y se convertiría en una expresión fácilmente disponible (readily available expression, cf. Slobin, 2006: 68). Aparte de la alta frecuencia hay otro testimonio que apoya lo dicho: la prueba que realizamos en el corpus Araneum Hispanicum Maius. Mediante la herramienta que facilita la búsqueda de unidades candidatas a colocaciones intentamos explorar el contexto derecho del lema salir (tres posiciones). Según la medida de asociación logDice, ${ }^{25}$ la palabra corriendo aparece en el tercer puesto de la lista generada. ${ }^{26}$ No observamos nada parecido en los otros verbos principales más habituales (ir, venir, llegar).

\section{Conclusiones}

En este texto nos hemos centrado en un caso especial de expresión de movimiento en español que comprende el uso de un verbo de movimiento en combinación con un gerundio de modo. Tras exponer los supuestos teóricos de la semántica cognitiva sobre los «eventos de movimiento» realizamos una serie de consultas en distintos corpus de textos españoles para averiguar qué formas participan frecuentemente en dicha construcción y si varía su uso en textos traducidos frente a textos originales.

Los datos indican que las expresiones de MANERA mediante el gerundio de modo corriendo y volando son más frecuentes en textos traducidos. Nuestra interpretación de las divergencias en los dos casos es que, siendo eventos que describen acciones más dinámicas, los traductores las perciben como más marcadas y por eso prefieren mantener la información sobre la MANERA, incluso a costa de tener que utilizar un recurso que requiere un mayor esfuerzo cognitivo.

movimiento entero. En términos del marco cognitivo (cf. p. ej. Lakoff, 1987) hablaríamos de la «categoría» de verbos de manera de movimiento. Por un lado, volar, por ejemplo, sería el prototipo de movimiento utilizado cuando nos referimos a aviones o aves, pero probablemente se marcaría si estuviéramos hablando del ser humano, ya que dicho movimiento está muy alejado de su forma de moverse prototípica. Por otro lado, el movimiento de correr será menos marcado en situaciones en las que, por ejemplo, tenemos prisa, mientras que será más marcado cuando se utiliza en situaciones en las que es habitual caminar (p. ej. un paseo por la ciudad). En otros casos, sin embargo, caminar puede ser más marcado, por ejemplo, en contraste con el uso del transporte público (p. ej. ¿Por qué has tardado tanto? He venido caminando.) Suponemos también que la decisión del traductor de marcar o no marcar la MANERA puede verse influida por la «cantidad» o relevancia del contexto que ayuda a codificar la situación, puesto que, por ejemplo, si un pájaro sale del nido es muy probable que sea volando.

24 Por otro lado, también cabe considerar que podría tratarse del proceso de gramaticalización (cf. Nieuwenhuijsen, 2020). Un estudio futuro que analice el comportamiento tanto semántico como sintáctico de las unidades en cuestión podría revelar más sobre el supuesto proceso.

25 Una medida de asociación estadística que ayuda a detectar las colocaciones, identificando las más típicas. Se basa en la frecuencia de la base (node), el colocado y la frecuencia de la unidad entera.

26 Realizamos la prueba también en el segmento español de InterCorp, ver. 13, y los resultados eran muy parecidos: corriendo aparecía en el segundo puesto. 
Argumentamos también que en el caso de salir corriendo, cuya incidencia supera la de los otros, incluso podría tratarse como una unidad en vías de fraseologización.

\section{Bibliografía}

У ALONSo, Amado (1961). Estudios lingüísticos. Temas españoles. 2aㅡ. ed. Madrid: Gredos.

$\checkmark$ ASKE, Jon (1989). «Path predicates in English and Spanish: A closer look». Annual Meeting of the Berkeley Linguistics Society 15.1, pp. 1-14.

У BENKo, Vladimír (2015). Araneum hispanicum maius, verze 15. 04. Ústav Českého národního korpusu FF UK. http://www.korpus.cz [12-01-2021].

У CIFUENTES FÉREZ, Paula (2012). «La semántica conceptual». In: Iraide Ibarretxe-Antuñano; Javier Valenzuela (coords.). Lingüística Cognitiva. Barcelona: Anthropos, pp. 189-211.

У CIFUENTES FÉREZ, Paula; ROJO LÓPEZ, Ana (2015). «Thinking for translating: A think-aloud protocol on the translation of manner-of-motion verbs». Target 27.2, pp. 273-300.

\ CORPAS PASTOR, Gloria (1996). Manual de fraseología española. Madrid: Gredos.

У CROFT, William A., et al. (2010). «Revising Talmy's typological classification of complex event constructions». In: Hans C. Boas (ed.). Constructional Approaches to Language. Amsterdam; Philadelphia: John Benjamins Publishing Company, pp. 201-236. https://doi.org/10.1075/cal.10.09cro

У CUARTERo otAL, Juan (2006). «¿Cuántas clases de verbos de desplazamiento se distinguen en español?» Rilce. Revista de Filología Hispánica 22.1, pp. 13-36.

ע CVRČEK, Václav (2019). Calc: Corpus Calculator. Czech National Corpus. www.korpus.cz/calc [17-01-2021].

y ČERMÁK, Petr; VAVŘín, Martin (2020). Korpus InterCorp - španělština, verze 13. Ústav Českého národního korpusu FF UK. http://www.korpus.cz [17-01-2021].

У FERNÁNDEZ LAGUNILla, Marina (1999). «Las construcciones de gerundio». In: Violeta Demonte; Ignacio Bosque (dirs.).Gramática descriptiva dela lengua española, Vol.2. Madrid: Espasa Calpe, pp.3 443-3 503.

У FILIPović, Luna (2008). «Typology in action: applying typological insights in the study of translation». International Journal of Applied Linguistics 18, pp. 23-40. http://dx.doi.org/10.1111/j.1473-4192.2008.00189.x

$\checkmark$ IBARRETXE, Iraide (2003). «What translation tells us about motion: a contrastive study of typologically different languages». International Journal of English Studies, 3.2, pp. 151-176.

$\checkmark \quad$ LAKofF, George (1987). Women, Fire, and Dangerous Things: What Categories Reveal about the Mind. Chicago: Chicago University Press.

y MARTinkovÁ, Michaela (2018). «K tzv. sémantické typologii jazyků: Co česká slovesa pohybu mohou vypovídat o angličtině a španělštině». Studie $z$ aplikované lingvistiky 9.2, pp.37-53.

ע NÁdvorníkovÁ, Olga, et al. (2020). «The Romance Gerund and its Czech Respondents». In: Petr Čermák, et al. (eds.). Complex words, causatives, verbal periphrases and the gerund: Romance languages versus Czech (a parallel corpus-based study). Praha: Karolinum Press, pp. 107-146.

$\checkmark \quad$ NIEUwenhuijSEn, Dorien (2020). «El papel de las unidades convencionalizadas en los procesos de gramaticalización. El caso de la perífrasis andar buscando». In: Mar Garachana (ed.). La evolución de las perífrasis verbales en español. Una aproximación desde la gramática de construcciones diacrónica. Berlin: Peter Lang, pp. 241-266.

У REAL ACADEMIA ESPAÑola (2016). Nueva gramática de la lengua española (libro electrónico) [NGDLE]. Madrid: Espasa Libros.

y ReAl ACAdEMia española (2021). Corpus de Referencia del Español Actual. Versión 0. 3. [CREA]. Real Academia Española. https://apps.rae.es/CREA/view/inicioExterno.view [17-01-2021]. 
У ROSEN, Alexandr; VAVŘíN, Martin; ZASINA, Adrian Jan (2020). Korpus InterCorp, verze 13. Ústav Českého národního korpusu FF UK. http://www.korpus.cz [10-01-2021].

У SLOBIN, Dan Isaac (1996). «From "thought and language” to “thinking for speaking”». In: John J. Gumperz, et al. (eds.). Rethinking linguistic relativity. Cambridge: Cambridge University Press, pp. 70-96.

$\checkmark$ SLOBIN, Dan Isaac (2004). «The many ways to search for a frog: linguistic typology and the expression of motion events». In: Sven Strömqvist, et al. (eds.). Relating events in narrative, Vol. 2. Typological and contextual perspectives. New Jersey: Lawrence Erlbaum Associates, pp. 219-257.

У SLOBIN, Dan Isaac (2005). «Relating narrative events in translation». In: Dorit Ravid, et al. (eds.). Perspectives on language and language development: Essays in honor of Ruth A. Berman. Boston: Kluwer Academic Publishers, pp. 115-129.

У SLOBIN, Dan Isaac (2006). «What makes manner of motion salient? Explorations in linguistic typology, discourse, and cognition». In: Maya Hickmann, et al. (eds.). Space in languages: Linguistic systems and cognitive categories. Amsterdam; Philadelphia: John Benjamins Publishing Company, pp. 59-81. SLOBIN, Dan Isaac; HoITING, Nini (1994). «Reference to movement in spoken and signed languages: typological considerations». Annual Meeting of the Berkeley Linguistics Society 20.1, pp. 487-505. https:// doi.org/10.3765/bls.v20il.1466

У TALMY, Leonard (1985). «Lexicalization patterns: semantic structure in lexical forms». In: Timothy Shopen (ed.). Grammatical categories and the lexicon. Language typology and syntactic description. Cambridge: Cambridge University Press, pp. 57-149.

$\checkmark$ TALMY, Leonard (1991). «Path to realization: A typology of event conflation». Annual Meeting of the Berkeley Linguistics Society 17.1, pp. 480-520. https://doi.org/10.3765/bls.vl7i0.1620

$\checkmark$ TALMY, Leonard (2000a). Language, speech, and communication. Toward a cognitive semantics, Vol. 1. Concept structuring systems. Cambridge (Mass.): The MIT Press.

$\checkmark$ TALMY, Leonard (2000b). Language, speech, and communication. Toward a cognitive semantics, Vol. ll: Typology and process in concept structuring. Cambridge (Mass.): The MIT Press.

У TESNIÈRE, Lucien (2015). Elements of Structural Syntax [traducido por Timothy Osborne y Sylvian Kahane]. Amsterdam: John Benjamins.

$\checkmark \quad$ YLLERA, Alicia (1999). «Las perífrasis verbales de gerundio y participio». In: Violeta Demonte; Ignacio Bosque (dirs.). Gramática descriptiva de la lengua española, Vol. 2. Madrid: Espasa Calpe, pp. 3390-3 441.

$\checkmark \quad$ VINAY, Jean-Paul; DARBELNET, Jean. (1958). Stylistique comparée du français et de l'anglais: Méthode de traduction. Paris: Didier.

У WHITLEy, Melvin Stanley; GONZÁLEz, Luis. (2015). Gramática para la composición. $3^{\mathrm{a}}$ ed. Washington: Georgetown University Press.

\author{
Petr Šlechta \\ Katedra romanistiky \\ Filozofická fakulta \\ Ostravská univerzita \\ Reální 5 \\ 70103 OSTRAVA \\ República Checa
}

